\title{
La politique des droits en prison
}

Police institutionnelle, militantisme juridique, luttes dÉmocratiques

\section{Gilles Chantraine et Dan Kaminski}

\section{(2) OpenEdition}

\section{Journals}

Édition électronique

URL : http://journals.openedition.org/champpenal/2581

DOI : $10.4000 /$ champpenal.2581

ISSN : $1777-5272$

Éditeur

Association Champ pénal / Penal field

Ce document vous est offert par Université de Lille

\section{Université de Lille}

\section{Référence électronique}

Gilles Chantraine et Dan Kaminski, «La politique des droits en prison », Champ pénal/Penal field [En ligne], Séminaire Innovations Pénales, mis en ligne le 27 septembre 2007, consulté le 10 janvier 2020 URL : http://journals.openedition.org/champpenal/2581; DOI : 10.4000/champpenal.2581

Ce document a été généré automatiquement le 10 janvier 2020

(c) Champ pénal 


\title{
La politique des droits en prison
}

\author{
Police institutionnelle, militantisme juridique, luttes dÉmocratiques
}

\section{Gilles Chantraine et Dan Kaminski}

Introduction : droit(s) et politique en prison

1 Notre contribution ${ }^{1}$ vise à décrire les changements intervenus ou attendus dans le domaine pénitentiaire sous l'effet de la promotion et de la reconnaissance relatives de droits aux détenus, afin de saisir leurs potentialités innovatrices. Peut-on démocratiser une institution structurellement anti-démocratique? Quelle est l'efficace de l'introduction du droit dans un univers où les sujets sont privés de liberté ? Ces questions lancinantes ne font qu'actualiser une ambivalence maintenant classique qui caractérise les critiques de la prison, structurent ses stratégies de lutte, cimentent ses oppositions internes (réelles ou de principe) et aiguisent ses efforts réflexifs. Cette ambivalence se loge d'abord au creux de la question du statut sociohistorique des réformes carcérales : participent-elles à la réduction de l'indignité consubstantielle à l'enfermement forcé ou, sous couvert et par le biais même de cette transformation, participe-t-elle avant tout à la consolidation de l'institution, à la recherche d'une stabilité nouvelle obtenue grâce la neutralisation des critiques dont sont l'objet cette irréductible indignité ? L'ambivalence retombe sur un « dilemme des prisons » maintenant classique, rappelé avec clarté par Y. Cartuyvels $(2002,130)$ :

D'un côté, chercher à faire de la prison un espace de droit, c'est entériner l'existence d'un espace carcéral qui souffre d'un grave déficit de légitimité, accepter le maintien d'un système pénal qui repose sur une privation de liberté qu'on peut juger contraire aux droits de l'homme, accepter la violence d'une logique discriminatoire qui gère l'exclusion (...) de l'autre, refuser toute réforme de la prison, c'est s'empêcher de penser le développement d'un droit de l'exécution des peines, source de garanties, de protection et d'améliorations véritables pour les détenus confrontés, dans leur vie quotidienne, à l'arbitraire et au vide du monde carcéral.

2 L'innovation, boussole du projet qui a abouti à cette publication collective, prend ici valeur de concept heuristique et tactique afin d'objectiver ce qu'elle n'est pas, soit l'univers des changements observables mais non nécessairement «innovateurs ». L'innovation est en ce sens une autre manière de poursuivre l'exploration analytique de l'inertie historique 
lourde qui caractérise les prisons. L'innovation, pour le dire autrement, est une théorisation « exigeante » du changement, en ce sens qu'elle réfute le statut d'innovation à tout un ensemble de simples transformations qu'une autre focale théorique érigerait peut-être en changement significatif, voire de premier ordre. Il faut donc saisir l'innovation comme un concept à la fois heuristique et critique qui permet d'observer empiriquement l'ensemble des transformations sociales de l'institution tout en introduisant une distinction essentielle relative aux changements réels portés par ces transformations. Ce point de vue est clarifié par J.-F. Cauchie, lorsqu'il distingue une reproduction redondante (ou "à l'identique ») du système pénal (lorsque l'adaptation du système à son environnement est strictement conforme à la rationalité dominante qui le sous-tend) d'une reproduction innovatrice, qui «se caractérise par l'émergence d'un élément inattendu et imprédictible par rapport à la rationalité pénale moderne dominante, ou par rapport aux changements normaux dans la structure normative du système » (Cauchie, 2005, 412).

3 Dans la continuité de cette première distinction et arrimés à l'analyse de situations sociohistoriques concrètes, nous verrons cependant que l'enjeu critique n'est pas tant de constater ou de nier l'innovation. En effet, au regard de la définition proposée, un constat princeps, tout aussi bien théorique qu'empirique, s'impose : la promulgation et l'octroi de droits aux reclus dans une institution totale sont une innovation formelle ${ }^{2}$. Les «droits des détenus " mobilisent une rhétorique et tendent à instaurer des pratiques qui vont partiellement à l'encontre de la rationalité dominante du système, le détenu-commecitoyen entrant en collision avec l'opposition entre le "criminel» et "l'honnête homme ». Cette dernière opposition est une pratique divisante (Foucault, 2001 [1982], 1042) typique d'une rationalité «qui accepte certainement les principes de la dignité de l'adversaire, mais qui ne représente pas moins le transgresseur pris dans le filet comme un « Objet-Ennemi » (Pires, 1998, 6).

4 Mais force est de constater, et c'est là toute l'ambiguïté qui pose d'emblée l'inachèvement irréductible de la question, que cette collision discursive s'est opérée à l'intérieur même d'un espace guerrier-défensif, épure incarnée de cette même rationalité (Chantraine, 2006a). En d'autres termes, par l'introduction des «droits des détenus", la prison et le système de pensée qui la sous-tend se sont en quelque sorte "enrichies » d'une sousrationalité interne qui constate et amoindrit partiellement les « excès » de sa rationalité dominante. L'émergence d'un élément divergent de la rationalité du système institue ainsi un principe de limitation du caractère tyrannique et despotique de l'institution (voir Chauvenet, 2006). Elle intègre, à l'intérieur même de sa rationalité, une forme de pensée sur et contre ses « excès »: « limiter les effets néfastes de l'emprisonnement » dans une institution dont on connaît le caractère suicidogène, "maintenir les liens familiaux » dans une institution qui les brise, etc.

5 En conséquence, l'innovation en matière carcérale constitue un support d'analyse de la collision de rationalités inégales qui ensemble sous-tendent les modalités complexes de régulation des comportements en détention. L'enjeu consiste donc moins à déceler l'existence ou l'inexistence de l'innovation qu'à examiner le caractère plus ou moins périphérique ou plus ou moins central d'une "nouveauté », autrement dit à examiner la manière dont sa confrontation à des pratiques "traditionnelles" galvanise, ampute ou annihile sa portée, la réduisant dans ce dernier cas à une simple "potentialité innovatrice ». Bien que partiellement stabilisée, l'innovation est toujours susceptible de 
se réduire à l'état de "virtualité ». Il s'agit donc d'explorer l'ampleur concrète de la limitation par le droit du caractère intrinsèquement tyrannique de la prison.

Dans ce cadre, et au regard du dilemme sociopolitique des prisons énoncé supra, nous opérerons un premier retournement en abordant le problème sous l'angle d'une sociologie de la critique : la critique sociale de la prison sera appréhendée comme l'un des moteurs essentiels des transformations de l'institution. Il s'agira donc d'examiner la façon dont l'institution pénitentiaire s'est transformée en intégrant, contournant, domestiquant ou en désamorçant l'ensemble polymorphe des critiques dont elle a été et dont elle est l'objet. La scandalisation des conditions d'emprisonnement procède de voies diverses, qui vont de la publication d'un ouvrage-brûlot, tel celui du docteur Vasseur (2000) aux mouvements de révolte; ces derniers, aussi rares soient-ils, bouleversent les prisons et l'opinion publique. Or ces bouleversements se traduisent aujourd'hui massivement dans le langage du droit, et de la promotion des droits des détenus. Cela a été le cas en France en 2000 (projet d'élaboration d'une loi pénitentiaire abandonné sous la pression colonisatrice du « débat » sur la lutte contre l'insécurité) puis en 2006-2007 lors des "États généraux de la condition pénitentiaire ". Il y aurait en effet un long travail à faire pour comprendre, les conditions de la mise sur l'agenda politique d'une réforme visant à promouvoir substantiellement les droits des détenus. Ici, il s'agira plus modestement, de "piocher» dans l'histoire récente des prisons occidentales (et d'abord en France, en Belgique et au Canada) pour saisir comment différentes formes d'action politique ont contribué à réorganiser l'économie des rapports sociaux en détention. Cette fragmentation assumée de l'analyse fait de notre article une contribution exploratoire qui vise d'abord à susciter d'autres recherches à la fois plus ciblées et plus abouties en sociologie de la politique des droits en prison.

7 En nous inspirant des travaux philosophiques de J. Rancière, nous appréhenderons les modes de régulation des conduites en prison sous le vocable de la police institutionnelle, la " police » désignant l'ensemble des processus par lesquels s'opèrent l'agrégation et le consentement des collectivités, l'organisation des pouvoirs, la distribution des places et fonctions et les systèmes de légitimation de cette distribution (Rancière, 1995) ${ }^{4}$. La police, dit J. Rancière, est :

un ordre du visible et un ordre du dicible qui fait que telle activité est visible et que telle autre ne l'est pas, que telle parole est entendue comme du discours et telle autre comme du bruit. C'est par exemple une loi de police qui fait traditionnellement du lieu de travail un espace privé non régi par les modes de voir et du dire propres à ce que l'on appelle l'espace public, où l'avoir part du travailleur est strictement défini par la rémunération de son travail. La police n'est pas tant une "disciplinarisation" des corps qu'une règle de leur apparaître, une configuration des occupations et des propriétés des espaces où ces occupations sont distribuées (Rancière, 1995, 52-53).

Dans la libre continuité du philosophe, la police institutionnelle désignera pour nous à la fois les régimes de gouvernement et l'art gouvernemental d'une institution pénitentiaire et les rationalités et les systèmes de légitimation qui sous-tendent cet art et ces régimes. La police institutionnelle carcérale, en tant qu'art gouvernemental, caractérise l'organisation du sensible carcéral et le processus de dépolitisation qui lui est intrinsèque. Dépolitisation parce que J. Rancière oppose au concept de police ceux de politique et de démocratie - le second constituant l'essence du premier - saisies non pas comme un style de vie sociale ou un régime politique, mais comme le mode de subjectivation par lequel existent des sujets politiques. Faisant référence à la source grecque de la démocratie, J. 
Rancière nous rappelle que le démos grec, avant d'être le nom de la communauté tout entière, était le nom d'une partie de cette communauté : les pauvres; pas simplement défavorisés économiquement, mais ceux qui n'étaient pas comptés - au sens littéral du terme - pour avoir droit à la parole. C'est cette anomalie originaire qui définit le mieux le processus démocratique, quand ceux qui n'ont pas droit à la parole parlent, quand ceux qui sont hors-champ surgissent sur la scène de la politique.

Je propose maintenant de réserver le nom de politique à une activité bien déterminée et antagonique à la première : celle qui rompt la configuration sensible où se définissent les parties et les parts ou leur absence par une présupposition qui n'y a par définition pas de place : celle d'une part des sans-part. Cette rupture se manifeste par une série d'actes qui refigurent l'espace où les parties, les parts et les absences de parts se définissaient. L'activité politique est celle qui déplace un corps du lieu qui lui était assigné ou change la destination d'un lieu ; elle fait voir ce qui n'avait pas lieu d'être vu, fait entendre un discours là où seul le bruit avait son lieu, fait entendre comme discours ce qui n'était entendu que comme bruit. (...) Ce peut être celle de ces ouvriers du XIXe siècle qui mettent en raison collective des relations de travail qui ne relèvent que d'une infinité de rapports individuels privés. Ou encore celle de ces manifestants ou barricadiers qui littéralisent en "espace public» les voies de communication urbaines. Spectaculaire ou non, l'activité politique est toujours un mode de manifestation qui défait les partages sensibles de l'ordre policier par la mise en acte d'une présupposition qui lui est par principe hétérogène, celle d'une part des sans-parti laquelle manifeste elle-même, en dernière instance, la pure contingence de l'ordre, l'égalité de n'importe quel être parlant avec n'importe quel autre être parlant. Il y a de la politique quand il y a un lieu et des formes pour la rencontre entre deux processus hétérogènes. Le premier est le processus policier au sens que l'on a tenté de définir. Le second est le processus de l'égalité. Entendons provisoirement sous ce terme l'ensemble ouvert des pratiques guidées par la supposition de l'égalité de n'importe quel être parlant avec n'importe quel autre être parlant et par le souci de vérifier cette égalité (Rancière, 1995, 52-53).

Dans ce cadre, l'hypothèse première qui a initié la contribution présente était celle-ci : l'intrusion dans le système pénitentiaire "d'un élément inattendu et imprédictible par rapport à la rationalité pénale moderne dominante » - ce qui constitue, pour Cauchie et Kaminski (2007) l'un des nœuds problématiques de la question de l'innovation pénale est d'abord le produit dérivé d'une activité démocratique définie comme une entreprise de politisation de l'univers carcéral. Ainsi, cette contribution se proposait initialement d'examiner le rôle de la politique, soit, au sens de Rancière, de la prise de parole par les détenus eux-mêmes, dans la reconfiguration des modes de régulation institutionnelle des prisons.

10 À cet égard, la pétition, datée du 16 janvier 2006, signée par dix détenus condamnés à de longues peines de prison incarcérés à la maison centrale de Clairvaux, puis sortie clandestinement de détention et largement médiatisée ensuite, constitue un événement politique $^{5}$. En suivant la terminologie de Rancière, elle est un surgissement de la politique dans l'ordre policier, soit l'ordre du visible et du dicible. Un « nous » se forme et produit une parole là où il n'y avait auparavant que du silence ou du bruit. À travers une subjectivation singulière, les « emmurés vivants » rendent visible le tort spécifique dont ils sont l'objet - ce que ne permettent évidemment pas les différentes figures de la monstruosité relative aux auteurs de crimes graves, ni même l'argot pénitentiaire qui use simplement de la durée pour qualifier le détenu " longue peine » - et déstabilisent l'ensemble d'évidences avec lequel l'abolition de la peine de mort est le plus souvent envisagée. Donnons ici, à titre d'illustration, un large extrait de cette pétition : 
Nous, les emmurés vivants à perpétuité du Centre pénitentiaire le plus sécuritaire de France (...), nous en appelons au rétablissement effectif de la peine de mort pour nous. Assez d'hypocrisie! Dès lors qu'on nous voue en réalité à une perpétuité réelle, sans aucune perspective effective de libération à l'issue de notre peine de sûreté, nous préférons encore en finir une bonne fois pour toutes que de nous voir crever à petit feu, sans espoir d'aucun lendemain après bien plus de vingt années de misères absolues. À l'inverse des autres pays européens, derrière les murs gris de ses prisons indignes "la République des Lumières et des libertés" de 2006 nous torture et nous anéantit tranquillement en toute apparente légalité, "au nom du peuple Français". (...) À quoi servent les peines de sûreté qu'on nous inflige quand une fois leur durée dûment purgée on n'a aucun espoir de recouvrer la liberté ? (...) Après de telles durées de prison tout rescapé ne peut que sortir au mieux sénile et totalement brisé. En pareil cas, qui peut vraiment se réinsérer socialement ? En fait, pour toute alternative, comme avant 1981, ne nous reste-t-il pas mieux à trouver plus rapidement dans la mort notre liberté ? De surcroît, pour nous amener à nous plier à ce sort d'enterré vif, on nous a ces dernières années rajouté murs, miradors, grilles en acier et maintes autres contraintes (...) Aussi, parce qu'une société dite "démocratique" ne devrait pas se permettre de jouer ainsi avec la politique pénale visant à l'allongement indéfini des peines, selon la conjoncture, l'individu ou les besoins particuliers : à choisir, à notre mort lente programmée, nous demandons à l'État français, chantre des droits de l'homme et des libertés, de rétablir instamment pour nous tous la peine de mort effective. »

11 Il s'agirait alors d'explorer, à travers l'histoire des prisons occidentales, les conséquences, ou l'absence de conséquences, de ce type de subjectivation politique à travers l'histoire des prisons. Soulignons-le : cette forme de subjectivation est ici d'autant plus intéressante pour nous que la prise de parole collective reste à ce jour expressément interdite dans les prisons françaises; de même, lorsqu'elle est autorisée, via par exemple des comités de détenus (Canada, Suède), les questions de sécurité (soit la structure même de l'institution...) sont formellement évacuées de cet espace de liberté, alors qu'elle forme explicitement l'un des axes de la dénonciation des pétitionnaires ${ }^{6}$. Surtout, ce type de dénonciation s'appuie expressément sur les valeurs universelles des droits de l'homme pour asseoir et construire sa légitimité.

Cependant, il a fallu rapidement infléchir l'intuition initiale. En effet, certaines formes de prise de parole - i.e. de politisation - peuvent très bien ne pas s'exprimer dans le strict langage des droits, et trouver pourtant une réponse policière dans les termes du droit; en outre, certaines formes de refiguration policière peuvent combiner l'octroi de droits à des modalités de transformations institutionnelles qui l'amputent dans la pratique ; enfin, et surtout, l'efficacité d'une forme particulière de subjectivation politique réside souvent dans l'existence de relais (des militants qui exigent une réforme, des journalistes qui visibilisent l'intolérable, une Cour qui déplace une jurisprudence, etc.) qui pour Rancière n'accèdent pas au statut de "politique», mais font encore partie de la police. Nos observations nous ont donc conduit à complexifier le projet initial, et à nous interroger sur l'ambiguité intrinsèque du langage des droits en prison, et, d'un point de vue théorique, à remettre en question l'étanchéité et l'hétérogénéité radicales des concepts de politique et de police chez Rancière.

14 Dans ce cadre ainsi reformulé, nous rappellerons dans un premier temps les deux formes principales de critiques sociologiques généralement formulées à l'égard de la promotion des droits en prison: cette promotion serait incapable 1) de détruire la primauté sécuritaire de l'institution d'une part, 2) d'en finir avec sa vocation disciplinaire d'autre 
part. D'abord la critique de la soumission du droit à l'ordre sécuritaire. Ici, la loi pénitentiaire belge votée en 2005 sera prise comme exemple caractéristique de cette soumission: des privilèges sont transformés en droits formels, mais les exceptions justifiées par la sécurité en réduisent la portée. Ensuite, la critique de l'accompagnement par le droit de la reconfiguration pénologique de l'institution. Il s'agira là d'objectiver le tour de force qu'a réussi l'institution en intégrant la critique de la discipline à son propre fonctionnement (en "prenant mieux en compte l'individu») tout en renforçant et en actualisant sa vocation correctionnaliste à l'âge néo-libéral. Ces deux thèses, pertinentes à de maints égards, permettent de rappeler la grande capacité d'adaptation de l'institution à la critique. Elles insistent sur le fait que les déplacements de la police institutionnelle par le biais des luttes « au nom des droits » sont des déplacements somme toute secondaires, qui n'affectent qu'à la marge l'organisation sécuritaire et la vocation correctionnaliste de l'institution.

Dans un second temps, nous ferons l'hypothèse que malgré leur pertinence relative, ces critiques ne permettent pas d'appréhender qualitativement, aujourd'hui, les usages réels, par tous les acteurs du champ pénitentiaire (détenus, surveillants, militants, direction, instances de contrôle, etc.) du droit en détention. Il s'agira notamment d'explorer le continuum sociologique qui lie des causes militantes, des engagements et entêtements politiques et académiques, l'ouverture de fenêtres d'opportunité, des crises politiques (voir Vigour, 2004 ; Dobry 1992), des événements déclenchants ou la mise au jour de scandales, des condamnations par la cour européenne des droits de l'homme, etc. La mise en évidence de ce continuum nous conduira, répétons-le, à critiquer le concept de politique tel qu'élaboré par Rancière. Nous défendrons l'idée que la transformation de la police institutionnelle est le produit d'une dialectique complexe entre des formes de subjectivation politique des détenus et d'un relais qui œuvre en deçà de cette subjectivation, via les recours, la jurisprudence, la publicité, la dénonciation, la sensibilisation, etc. ${ }^{7}$. Nous appuierons notre exploration sur un entretien sociologique réalisé avec un juriste militant œuvrant au sein de l'observatoire International des Prisons (OIP), afin d'illustrer certaines dimensions du cause lawyering en matière carcérale, soit la manière dont des juristes peuvent mettre leurs compétences et leur expertise au service d'une cause, transformant le droit en arme militante. Bien que cet entretien soit isolé et aurait gagné à être croisé à d'autres types de données empiriques rappelons-le, cette contribution a valeur d'exploration - nous avons fait le pari que cet entretien illustrerait «par le bas » les mécanismes par lesquels organes supranationaux de contrôle, activité militante et résistances des détenus s'articulent, s'auto-alimentent et se structurent mutuellement.

Enfin, dans un troisième temps, nous rappellerons que cette activité politique est mue par un objectif utopique au regard du fonctionnement de l'institution totale: les droits des personnes incarcérées constitueraient le butoir inaliénable des principes sécuritaires de l'institution. On spéculera sur les implications de cette utopie, en montrant notamment qu'elle structure l'horizon pratique des luttes explorées dans notre second point : la revendication de droits agit comme un révélateur qui vient éclairer, en leur résistant, les impératifs disciplinaires et sécuritaires auxquels l'espace carcéral s'ordonne, et qui sans cesse le portent à excéder silencieusement la simple privation de liberté (Bérard, Chantraine, 2007, 50). Ainsi, d'un point de vue sociopolitique, la focale ainsi réglée se distingue tout autant de l'optimisme philanthropique de la réforme pénitentiaire qui voit dans tout changement un progrès significatif, que de la critique radicale qui campe $a$ 
priori sur l'option selon laquelle toute tentative d'amélioration de l'institution est une entreprise de consolidation d'un système barbare - cédant ainsi au douteux « mieux, c'est pire ». Il s'agit en quelque sorte de sortir par le haut du dilemme des prisons en échappant «à la fausse opposition entre critique cynique et célébration béate " (Linhart, Moreau de Bellaing, 2005, 298) par le biais d'un renouvellement des outils d'observation. Nous tenterons cette sortie d'une telle manière que la dichotomie qui fonde ce dilemme apparaisse trop simple, offrant finalement trop peu de prise à l'observation sociologique comme à l'action politique.

1. La vitalité nouvelle de l'institution : le droit « récupéré » par l'inertie pénitentiaire

Deux ensembles de critiques dénoncent la faible efficacité du droit à transformer l'institution carcérale. Le premier ensemble de critiques détaille comment l'obtention et l'introduction d'un droit en prison sont indubitablement perverties par le dispositif sécuritaire de l'institution, et ce même lorsque cette obtention s'inscrit dans le cadre d'une véritable loi pénitentiaire (telle qu'elle existe par exemple en Belgique, mais pas encore en France). Le second ensemble de critiques cible, dans la continuité des travaux de Foucault sur le correctionnalisme et la discipline carcérale ${ }^{8}$, la manière dont la promotion des droits, dont on aurait pu espérer qu'elle fasse désormais de la détention " une simple privation de la liberté d'aller et venir ", revitalise en la déplaçant cette vocation originelle. Le système correctionnel canadien fera là figure d'idéal-type: la promotion politique des droits des détenus reformule et intensifie les formes de l'enrôlement subjectif des détenus, outillant la vocation correctionnaliste de l'enfermement.

1.1. Droits des détenus et police carcérale

Deux réserves fondamentales ont affecté jusqu'ici la reconnaissance actuelle des droits des détenus : la limitation drastique de la portée des droits et la dépolitisation de la lutte tributaire du resserrement des droits sur les conditions de vie.

1.1.1. Des droits limités

19 La loi pénitentiaire belge, votée le 12 janvier 2005, dont l'entrée en vigueur n'est encore que partielle (Beernaert, 2007), présente deux caractéristiques réductrices des espérances transformatrices que certains ont placées en elle: 1) elle organise un régime de droits le plus souvent soumis à l'exception des enjeux pour la sécurité ; 2) elle fait une place stratégique majeure à une forme contractualisée de disciplinarisation des détenus, sous les noms de plan de détention et de plan de reclassement; l'objectif de ces outils est, moyennant la participation du détenu à leur confection, de concilier l'exécution de sa peine avec ses besoins, définis largement comme éducatifs, familiaux, sociaux, sanitaires. La cuirasse du discours pénologique réformateur présente, outre ses bonnes intentions et ses résultats, deux défauts majeurs qui affectent la portée et la performativité de sa critique. Le premier - la soumission des droits à l'impératif de sécurité - sera discuté ici. Le second - la reformulation du projet pénologique - sera exploré plus loin via l'exemple canadien.

Le constat classique contemporain est celui d'une sécurité menacée par les droits et de droits limités par l'impératif sécuritaire : des privilèges sont transformés en droits mais les exceptions légales à leur revendication les retransforme en privilèges. Les droits ne sont le plus souvent reconnus que dans un espace creusé au cœur d'un impératif de sécurité ; autrement dit, cet impératif constitue la norme fondamentale devant laquelle tout droit subjectif doit céder le pas. Si on assiste bien à l'introduction d'un nouvel instrument d'une politique publique, cette introduction ne modifie pas pour autant la 
finalité de la politique en question ; c'est dans la terminologie de Hall (1986, 1993, cité in Lascoumes, 2006), un changement de deuxième ordre. Une position conservatrice soutenue par exemple en France par Bouloc (2005), ne conçoit même pas l'intérêt de reconnaître plus avant des droits aux détenus, droits déjà bien suffisants, sans faire courir des risques à l'impératif de sécurité. Alors que ce parti a été pris en Belgique, via l'adoption d'une loi pénitentiaire, Pierre Reynaert, ancien directeur de prison belge, conclut avec pessimisme que l'octroi de droits ne réduit rien à la suprématie de la sécurité sur les droits: "Tout ce que la loi accorde d'une main, elle le retire de l'autre main. Chaque disposition contient sa réserve, son exception. Comment ne pas être frappé par l'image de ce droit emprisonné ? Sans doute faut-il retourner la perspective : plutôt que d'octroyer des droits aux détenus, il s'agit d'octroyer le droit à l'administration pénitentiaire de s'en écarter dès qu'un 'motif supérieur' l'exige » (Reynaert, 2004, 241). À cet égard, l'alternance ou la substitution d'un projet réhabilitateur avec un projet sécuritaire (Morgan, 1994, 109-110) fragilise et corrompt irrémédiablement la logique des droits.

21 Le bénéfice procédural - octroyer un droit de plainte et de recours; permettre l'accès à des juridictions - de la reconnaissance de droits est non négligeable mais il semble déjà perverti. Une directrice de prison, interviewée à l'occasion d'un conflit social, énonce avec justesse que la reconnaissance de droits aux détenus consiste en un progrès procédural, qui rendra plus transparente et donc plus fiable la prise de décisions notamment en matière disciplinaire. D'autres directeurs de prison, en Belgique, ont récemment signé un texte dans lequel ils évoquent les décisions du conseil d'État cassant des sanctions disciplinaires : «fondamentalement les pratiques ne changent pas et c'est bien là le (...) problème: nous avons quand même glané quelques informations pour mieux formaliser ces décisions. Et nous leur donnons une apparence de légalité qui ne fait que dissimuler des pratiques qui, fondamentalement, n'ont pas changé et qui relèvent toujours plus du pouvoir du prince, que d'une procédure contradictoire et équilibrée » (de Thier et al., 2005).

22 Les droits des détenus peuvent aussi être interprétés comme une victoire ou encore le bénéfice ultime d'une révolution pénitentiaire (Herzog-Evans, 2002). En effet, pendant près de deux siècles, l'humanisme réformateur a « fait silence sur les droits » (Pires, 1992, 81). Mais du point de vue de la police de l'institution, on peut aussi concevoir qu'il s'agit d'une adaptation nécessaire aux exigences croissantes d'un principe de normalisation qui parcourent l'ensemble de la société (cela va des droits de l'enfant à ceux des homosexuels en passant par les débats sur les dispositions anti-discriminatoires à l'égard des étrangers ou des immigrés, voire la discrimination positive à leur égard). Bien que les enjeux et les conditions soient différenciés selon les secteurs du droit et les groupes concernés, cette lame de fond de la normalisation pèse sur l'État engagé par les droits de l'homme et garant de leur effectivité. Le recours aux droits subjectifs accordés à la minorité détenue peut apparaître en ce sens comme l'instrument d'une politique d'évitement du blâme ( avoiding blame politics : voir Lascoumes, 2006, qui emprunte l'expression à Weaver, 1986).

23 À cet égard, la terminologie de Hall, déjà évoquée, attribuerait sans doute à la reconnaissance des droits, le statut de changement de troisième ordre ou de changement de paradigme, tant on peut dire que c'est le domaine des finalités qui se modifie avec l'introduction de nouveaux instruments. La lecture cynique de l'évolution peut alors être la suivante. En suivant Pires, c'est dans un contexte de perte de légitimité de l'institution et de « désenchantement de la réforme pénitentiaire ( (Pires, 1992) que le discours des 
droits apparait, non seulement comme force d'opposition au modèle normatif discrétionnaire de la prison, mais aussi comme source de légitimité nouvelle pour l'institution. La lecture de l'introduction des droits comme changement de finalité (du soutien d'objectifs multiples et successifs de l'incarcération vers l'objectif du respect des droits des détenus), dépend tout autant de l'impasse d'un discours sur les objectifs que de la promotion positive des droits subjectifs, la légitimité de l'institution est dès lors préservée par sa prétention réduite à protéger le public tout en respectant les droits des détenus.

1.1.2. Reconnaissance des droits et dépolitisation des conditions de vie

Le besoin d'analyser les changements produits dans la normativité carcérale depuis les années 1970 tient dans l'échec relatif ou la percée relative de la critique : les révoltes (comme celles de Louvain par exemple; voir Mary, 1988), les actions militantes (du GIP par exemple ; voir Artières et al., 2003) ont eu leurs effets positifs sur les conditions de vie en prison, sans avoir atteint pour autant leur horizon. Dans les épreuves carcérales des années 1970, ce n'est pas en tant que victime que le détenu est perçu, mais comme sujet et acteur d'une histoire politique déniée et rendue inaudible. Foucault (1994a [1979]) est explicite : il fallait faire entrer la prison dans l'actualité, non sous la forme d'un problème moral, ou d'un problème de gestion générale, mais parce qu'il s'agit d'un lieu où il se passe de l'histoire, du quotidien, de la vie, des événements du même ordre qu'une grève dans un atelier, un mouvement de revendication dans un quartier, une protestation dans un HLM.

Mary (1988) montre comment suite à des révoltes carcérales, et notamment celle qui s'est déroulé à Louvain en 1976, la thématique des droits des détenus s'est éveillée puis resserrée autour de questions de procédures et de conditions de vie en prison. Questions essentielles certes, mais cette concentration a exclu du champ des réformes les droits qui devraient logiquement être indexés à la resocialisation des détenus, soit notamment les droits de participation à la vie carcérale, que l'on peut rassembler sous l'enjeu de démocratisation de la prison. Le spectre des droits promus et reconnus est souvent minimaliste, en raison du fait que le minimum vital n'est pas souvent atteint par la prison. En quelque sorte les besoins primaires de la (sur)vie l'emportent toujours sur les conditions de la constitution du sujet politique (un luxe néfaste pour l'administration et un luxe - non prioritaire, bien que revendiqué dans les révoltes carcérales - pour le détenu). En effet, quels qu'en soient les résultats, la révolte auto-constitue le détenu en sujet et les détenus en sujet collectif : un mouvement social s'y révèle avec ses dimensions organisationnelles et revendicatrices. La prise de parole est intrinsèquement politique, donc révolutionnaire au regard de l'institution totale. Il aboutit cependant à l'endogénéisation de la revendication, soit sa réinscription dans la logique pérenne de la prison, ou au recyclage de la révolte dans un changement de surface (l'amélioration relative des conditions de vie au détriment de la reconnaissance d'un nouveau sujet politique).

Ce que Philippe Mary nomme « l'aporie du changement pénitentiaire » nous renvoie à la fois au paradoxe du scandale (défini comme écart publiquement exposé entre les normes et leur effectivité) et aux enjeux heuristiques de l'innovation. Paradoxe du scandale, considéré comme une épreuve, événement à l'issue indéterminée : «le moment où est révélée publiquement l'existence d'une contradiction entre idéal normatif et pratiques est aussi celui où il redevient possible de faire disparaitre une telle contradiction " (Comité éditorial, 2005, 4). Enjeux heuristiques de l'innovation: celle-ci apparaît plus 
dans les processus de révolte et de lutte que dans les résultats qui lui sont attribuables, réduits, discrets, discontinus, décevants. Probablement faut-il reconnaitre ici même le sort dialectique commun (et non spécifique à la prison) du rapport établi par J. Rancière, entre politique et police, soit la capacité infinie de l'institution à endogénéiser les effets de la révolte comme la capacité infinie de la politique (illustrée ici par la révolte) à troubler la police et à l'obliger à se réaménager fût-ce partiellement. L'endogénéisation constitue paradoxalement la preuve que le scandale «ne laisse jamais les choses en l'état» (De Blic, Lemieux, 2005, 11). Cependant, le scandale de la prison est toujours contenu dans un « périmètre sectoriel » (De Blic, Lemieux, 2005, 36) qui se trouve en fin de comptes "renforcé dans son autonomie", d'autant plus que le statut politique de l'initiateur du scandale est, dans ce périmètre, disqualifié par le master status (Hughes, 1975) de criminel.

Ainsi, il semble incontestable que la poussée du droit en prison, et, surtout, l'ouverture de canaux de plaintes externes et le renforcement d'instances de contrôle externe, constituent progressivement un outil de protection contre les abus de pouvoir d'une administration désormais moins souveraine, et c'est là une réelle nouveauté. Mais cette poussée n'a pas tant, comme l'espéraient les abolitionnistes pragmatiques, contribué à mettre en œuvre des réformes qui, tout en améliorant les conditions de détention, mettraient l'institution face à son impossibilité démocratique et participeraient ainsi à son démantèlement progressif, qu'il n'a pas désarmé la critique de l'une de ses cibles traditionnelles et permis à l'institution de renforcer la légitimité de son dispositif (Chantraine, 2006a ; Kaminski, 2002).

\subsection{L'empowerment, les droits et le projet pénologique}

Le second obstacle affectant la potentialité innovatrice de la reconnaissance des droits des détenus réside dans le dispositif pénologique de la prison. À cet égard, le système correctionnel canadien fait figure d'illustration idéal-typique et nous décrirons ici la manière dont les transformations de la vocation correctionnaliste des pénitenciers canadiens a permis, pour une part, «d'absorber » la lutte pour les droits dans un projet renouvelé. Après avoir rapidement présenté les mécanismes par lesquels l'émergence d'un correctionnalisme "positiviste » et "scientifique», outillé par des savoirs-risques érigé en nouvelle religion pénologique, a pris forme au sein des services correctionnels canadiens, nous nous focaliserons essentiellement sur les prisons de femmes, et la manière dont des luttes féministes intellectuellement structurées autour de la notion d' empowerment ont contribué à reconfigurer la police de l'institution.

Au sein des pénitenciers canadiens ${ }^{9}$, l'émergence puis l'omniprésence de l'outil-risque doit être saisie comme une réponse institutionnelle à une double crise de légitimité. Crise de l'objectif de réhabilitation du système pénal d'abord. De fait, la critique rassemblait, sur la base d'arguments et d'idéologies contradictoires, marxistes, foucaldiens, conservateurs et libéraux (Cohen, 1985) : la réhabilitation est une illusion qui masque la violence des rapports de classe, la réhabilitation est le raffinement moderne de la cruauté, la réhabilitation s'illusionne quand elle croit supplanter les vertus sociales de la punition véritable, la réhabilitation coûte trop cher au regard de ses piètres résultats, etc. Crise d'une pratique thérapeutique clinicienne ensuite, à la fois dénoncée pour son laxisme à l'égard d'individus dangereux et pour son incapacité à adopter une « démarche scientifique efficace ». Cette double crise a constitué le support négatif d'une vaste réarticulation portée par la révolution cognitive, la multiplication des outils actuariels d'évaluation clinique et la responsabilisation accrue des bénéficiaires de l'intervention 
thérapeutique (Quirion, 2006), au cours de laquelle la pratique thérapeutique a cherché (et a trouvé) une nouvelle légitimité en réorganisant ses techniques de vérités.

Dans ce cadre, un processus formel alliant l'évaluation de facteurs de « risque de récidive " à une planification d'intervention visant à gérer le contenu correctionnaliste de l'enfermement ainsi qu'à organiser les modalités d'obtention des libérations conditionnelles, a été mis en place au début des années 1980. Ce système repose sur l'hybridation d'une gestion actuarielle (statistique) des "risques » et de pratiques thérapeutiques d'inspiration cognitivo-comportementale, structurée autour de l'identification des « besoins » ou « facteurs criminogènes » dynamiques des détenus. En suivant Hannah-Moffat (2005), on peut ainsi observer comment l'alignement du « risque » de récidive sur les « besoins » du prisonnier contribue à l'appréhension du détenu comme un " sujet à risque évolutif » (transformative risk subject) qui est propice aux interventions thérapeutiques ciblées. Cet alignement permet ainsi de réaffirmer la nécessité de réintégrer le délinquant dans la communauté et de réduire les risques de récidive. L'inclusion des « besoins » du prisonnier dans le calcul du risque reconfigure les efforts interventionnistes et lie les stratégies de gestion du risque à des stratégies réhabilitatives sous-tendues par une théorie psychologique normative de la personne incriminée. En résumé, l'association risque-besoin et le mode de pensée hybride (clinique-actuariel) sur lequel elle repose forge le coeur d'un modèle correctionnaliste désormais néo-libéral (Chantraine, 2006b).

31 Le rapport intitulé La création de choix (voir Shaw, Hannah-Moffat, 2002 ; Frigon, 2002, Hannah-Moffat, 2005) a approfondi l'esprit protecteur et émancipateur des droits des femmes détenues en s'appliquant à promouvoir des principes directeurs de l'incarcération des femmes: l'autonomisation de ces dernières par l'offre de programmes ; l'offre, par ces programmes de choix significatifs et d'opportunités réalistes de développement personnel des femmes, le traitement avec respect et dignité des femmes incarcérées, le développement d'un environnement carcéral de soutien, le développement de la responsabilité partagée.

Il est intéressant de noter la façon dont Sylvie Frigon évalue le caractère innovateur des travaux qui ont abouti au rapport La création des choix: "finalement, une logique de réforme et non de transformation triomphera lors de ces travaux et de leur implantation " (Frigon, 2002, 18-19). Kelly Hannah-Moffat utilisera la formule la plus lapidaire pour rendre compte des effets du rapport sur la "gouvernance pénale »: les femmes sont dorénavant incarcérées et incarcérées plus longtemps, dans l'objectif de les autonomiser (empowering). De tels constats reposent sur une limite inhérente à la transformation de l'expérience de l'incarcération fondée sur l'empowerment: en effet, la valorisation des besoins des détenues dans le dispositif pénitentiaire s'est mariée à la question de l'évaluation des risques. En quelque sorte l'absorption des valeurs par le système correctionnel entraîne un effet d'individualisation accrue du régime carcéral, mais « sous le couvert de l'autonomisation » (Hannah-Moffat, 2005).

33 La soumission du régime pénitentiaire à un objectif pénologique constitue donc un second obstacle à la valeur innovante des droits des détenus. Le néo-correctionnalisme constitue en quelque sorte la réponse de la prison à sa critique carcéralo-centrée. Un « Nouvel Esprit de la Pénologie » participe à la production du détenu capable (plutôt que coupable), lui-même produit dérivé d'une critique du système carcéral. Le néocorrectionnalisme conserve l'objectif du correctionnalisme mais use de leviers subjectifs nouveaux tels la responsabilisation et l'hypermotivation (soit l'enrôlement subjectif) du 
détenu. Il emprunte ainsi aux intuitions du management moderne qui prévaut, idéologiquement du moins ${ }^{10}$, dans de nombreux mondes professionnels. Le détenu peut aussi être « responsabilisé » dans son parcours de détenu et devenir le client, par contrat, d'une offre de services dans laquelle le sujet de droit ressemble plus à l'homme-lige (voir Kaminski, 2006) qu'au détenteur de droits subjectifs opposables à l'institution. L'un de nous a soutenu que la reconnaissance de droits aux personnes détenues "qui peut s'interpréter comme une victoire pour des catégories d'individus démunis consiste plus fondamentalement en un progrès du cynisme managérial, sous l'empire duquel l'État distribue mollement ses responsabilités, exprimées en termes de droits individuels; en échange, sans rien lâcher de sa vigueur répressive, l'État s'offre le luxe d'escamoter la question normative essentielle de la justification du recours à l'emprisonnement » (Kaminski, 2002, 91). Cet escamotage ne signifie pas spécifiquement la disparition de justifications portées par les discours publics mais bien plutôt leur accumulation inconséquente (de la neutralisation à la réparation en passant par la rétribution) ou leur caractère purement rhétorique, laissant au néocorrectionnalisme la place d'une évidence d'ordre méthodologique. On peut soutenir, après d'autres (O'Malley, 1992; Hannah-Moffat, 2000), que les droits constituent une ressource dans la stratégie néo-libérale complexe de récupération de l'empowerment. Il s'agit de gouverner les détenus à travers leur subjectivité, par le droit, plutôt que contre elle, par la discipline. L'horizontalité apparente du rapport juridique peut produire l'assujettissement mieux que sa conception disciplinaire, autoritaire et verticale. À la mesure de l'avancée du prudentialisme pénal, l'institution s'avance sur la voie de la reconnaissance des droits spécifiques aux détenus. Cette reconnaissance loin de constituer une rupture politique, produit une discrimination plus raffinée des méthodes de gouvernement adressées aux powerable et aux unpowerable: le nouveau sujet de droits qui ne saisit pas l'occasion de devenir un sujet prudent sera ainsi responsabilisé ; l'autre, qui n'atteindra pas la prudence requise continuera à être discipliné et à perdre les bénéfices des "avantages" acquis. On peut dès lors douter qu'en modifiant ses procédures et le statut juridique de ses clients, la prison change aussi de structure et de programme... Elle se diversifie plutôt, opérant un nouveau régime de classification avec la coopération des détenus... Le recours accru au levier « motivationnel » dans la gestion des parcours de détention (plans de détention et de reclassement en Belgique) et l'indexation de la «motivation » aux dimensions sécuritaires de l'ordre carcéral (voir par exemple Chantraine, Vacheret, 2005) réorganisent les contraintes carcérales autour de pratiques négociées, d'engagements et de collaborations.

On peut penser par ailleurs que les voies de recours instituées pour la revendication singulière de droits seront d'autant moins susceptibles de produire de l'innovation que la logique pénologique risque d'entraver la logique des droits ${ }^{11}$. Dunkel (1997) explique le faible taux de succès du droit de plainte en Allemagne par l'espace d'indétermination, favorable à l'administration, que laissent les textes reposant sur l'idéologie du traitement des détenus. L'indétermination (et la variabilité) politique des objectifs de l'emprisonnement permettent de réduire la portée et la nature des droits reconnus.

1.3. Une nuit où tout se vaut?

35 Ces deux critiques des droits des détenus ont l'avantage certain de forcer l'analyste à la vigilance et à mesurer toute la spécificité du droit en prison, miné par sa structure sécuritaire et sa vocation disciplinaire. Ce n'est pas parce qu'un droit est formulé qu'il sera garanti, et, même, le respect d'un droit particulier peut accompagner, parallèlement, l'exercice d'un pouvoir qui amputera un autre droit ailleurs. Mais ces critiques risquent 
aussi de tomber dans un travers majeur : celui d'être incapable d'observer avec toute la minutie nécessaire la reconfiguration des rapports sociaux en détention en cédant à un curieux nihilisme selon lequel toute transformation institutionnelle ne serait qu'un « mirage $»^{12}$ : on croit voir le pouvoir s'adoucir, c'est qu'en fait il avance mieux masqué, signe indubitable de sa perversion accrue. Or rien n'est plus faux ni plus contreproductif de faire de toute police, quelle qu'elle soit, « la nuit où tout se vaut » (Rancière, 1995, 53)

${ }^{13}$ : en prison comme ailleurs, il y a de la moins bonne et de la meilleure police :

Ce n'est pas parce qu'un droit en prison tend inexorablement à prendre place dans le fonctionnement de l'institution (c'est-à-dire à secréter sa part d'arbitraire), qu'il est indifférent. D'une part, il n'est pas indifférent que l'administration se donne comme objectif zéro évasion et la répression par la force de tout mouvement de protestation, ou qu'elle accorde des espaces de dialogues et renonce à la vision paranoïaque d'un risque permanent et omniprésent. Il n'est pas indifférent que les détentions soient gérées par la menace, voire par la violence pure et simple, ou par l'octroi de possibilités larges de travail, de formation, et de liens avec la famille. Il n'est pas indifférent que les détenu(e)s aient des perspectives de sortie, pour que les possibles offerts en détention aient un début de sens, plutôt que, condamnés à des peines de sûreté interminables, ils ne puissent, aux yeux de l'institution, qu'être gardés dans une situation de tension permanente. Il n'est pas non plus indifférent que le pouvoir discrétionnaire de l'administration soit pur et intact, ou que des instances indépendantes s'y immiscent pour faire valoir le point de vue du droit, même si cette tentative est vouée à demeurer imparfaite (Bérard, Chantraine, 2007, 51-52).

Surtout, si le droit reste soumis à l'ordre sécuritaire de la prison et semble parfois se réduire à un simple "fluidifiant» des rapports de pouvoir en détention, la nouvelle organisation carcérale offre néanmoins une meilleure prise aux luttes concrètes, qui viennent "gripper" cette stabilité retrouvée. La promotion des droits en prison transforme notamment le régime même de visibilité de l'institution. Close sur elle-même, organisée pour se cacher du monde extérieur derrière ses hauts murs tout en assurant la visibilité totale du détenu (panoptisme, œilleton), elle est désormais sous les feux des projecteurs des médias et de la société civile. L'un d'entre nous a rencontré, dans un pénitencier canadien, un ancien directeur qui lui confiait: «nous, les services correctionnels, aujourd'hui, on est comme un poisson dans un bocal».

2. Droit et politique : les connexions hétérogènes du contre-pouvoir

Pour Rancière, seule la subjectivation constitue véritablement l'essence de la politique, lorsque, pour le cas qui nous intéresse, les détenus prennent eux-mêmes la parole là où tout est fait pour en empêcher l'expression, et réclament, au nom d'un « nous » singulier, une transformation de leur condition. Par exemple, pour le philosophe, les révoltes de novembre 2005 en France ne seraient pas d'ordre "politique» parce qu'elles ne construiraient pas un "monde commun"; dans le même sens, la substitution de la revendication de « dignité » à celle d'« égalité » des sans-papiers, échouerait à constituer leur action en véritable action "politique ${ }^{14}$. Que dirait Rancière alors de ces militants qui réclament en France la promulgation d'une loi pénitentiaire? Face à cette ségrégation conceptuelle, qui fait de la notion de politique une notion quasi-métaphysique et radicalement anti-sociologique, nous voudrions au contraire explorer l'imbrication concrète des mécanismes de police et de politique, pour décrire la nouvelle donne carcérale, produit pour une part, de la promotion des droits des détenus.

L'exploration, on l'a annoncé, sera partielle. Nous avons choisi de nous appuyer sur le discours d'un militant-expert de l'OIP recueilli dans le cadre d'un entretien sociologique 
pour illustrer l'alimentation mutuelle de divers registres de luttes. Cette alimentation mutuelle devra faire par la suite l'objet d'études systématiques et approfondies, délestées d'une approche strictement juridique de la promotion des droits, et enracinées dans une sociologie du cause lawyering, une sociologie de la politique militante des droits en prison.

2.1. De la guérilla juridique

Pour notre interlocuteur, la promotion des droits n'est pas tant une fin en soi qu'un moyen en vue de deux objectifs généraux: "protéger les détenus » et « réduire l'arbitraire en détention ». L'usage militant du droit marque également un tournant dans l'histoire de l'OIP : passer d'une simple «dénonciation» de l'arbitraire, époque durant laquelle l'association était marquée par une certaine méfiance à l'égard du droit («le droit ne peut être qu'un instrument du pouvoir ") à l'action concrète qui retourne le droit en instrument de revitalisation des contre-pouvoirs. Là, d'autres associations, notamment de défense des étrangers (comme le Gisti par exemple) constituent des modèles en la matière : depuis de nombreuses années, des professionnels du droit (universitaires notamment) mettent leurs compétences au service de la défense des droits des étrangers, et font avancer dans leur propre champ la doctrine juridique sur la question. L'arme juridique n'est donc en rien idolâtrée, elle est mobilisée d'abord parce qu'elle permet d'attaquer l'institution là où elle présente le plus de faiblesses.

C'est un des points d'attaque de l'institution, sa fragilité au regard du droit, puisque fondamentalement elle fonctionne par la négation du droit des individus, et elle cherche constamment à avoir le dessus sur une personne. Donc forcément, ça a des conséquences : ça se traduit par une qualification juridique, donc la critique du fonctionnement de l'institution passe par là aussi.

La philosophie générale de cette activité militante est celle de la " guérilla »: la lutte est conçue comme fragmentée, opérant sur différents fronts simultanément (fouilles à corps, transfert arbitraires, etc.) et sous diverses formes qui se nourrissent mutuellement. Cette lutte opère en-deça de deux utopies. Utopie abolitionniste d'abord: la suppression totale et définitive de la prison n'est pas jugée accessible dans un futur proche. Utopie de la bonne gestion pénitentiaire ensuite, la «bonne prison»: la prison, dans son essence même, excèdera toujours la simple privation de liberté, les écarts aux droits existeront toujours. Mieux, les visions évolutionnistes et progressistes sont elles-mêmes rejetées : si le droit progresse dans différents domaines, il peut très bien régresser dans d'autres.

Il faut pas avoir une vision statique de la situation. L'administration est constamment en recherche de dispositifs nouveaux de contrôle, de sujétion, etc. On le voit avec ce qui s'est passé après 2002, l'intervention des ERIS, etc. Nous on essaie de faire valoir, dans le cadre de recours la dangerosité de ce type de dispositif... Et puis, il y a des régimes de sécurité qui sont de plus en plus contraignants qui ont été élaborés depuis 2002, essentiellement du temps de L..., le directeur de l'A. P. [administration pénitentiaire] Il y a parfois, on est dans une espèce d'endiguement et de la progression de l'arbitraire. Le droit progresse sous différents aspects, mais il y a aussi la tentation de l'A.P. d'aller vers d'autres choses, d'autres choses qui sont autrement plus dures [telles les maisons centrales de sécurité renforcée], qui sont censées être dans un premier temps ciblées à une catégorie spécifique de détenus, mais qui sont vraiment très dures...

Il s'agit alors, dans une lutte constamment renouvelée, de réduire les poches d'arbitraire là où elles sont les plus intolérables et/ou en fonction de l'état des forces et de "l'attaquabilité » juridique du phénomène, toutes ces dimensions se renouvelant en fonction de chaque nouvel acquis. À la question «qu'est-ce qui serait pour vous une innovation pénale?", notre interlocuteur, après réflexion et évocation d'une future loi 
pénitentiaire, pose trois éléments qui illustrent bien la pluralité des jeux d'échelle des luttes : l'abolition des fouilles corporelles, la mise en place d'un contrôle extérieur et indépendant, un accès massif au droit pour les détenus qui sont incapables d'y accéder seuls. L'optique rejoint ici l'exigence posée par trois directeurs de prisons belges, pour qui la reconnaissance des droits des détenus doit, compte tenu de la spécificité de l'institution, présenter des marqueurs d'effectivité particuliers : le droit en prison devrait être rapide (sans quoi il serait tout aussi rapidement inutile : le recours visant à empêcher une mesure de quartier disciplinaire doit intervenir... avant la mise en quartier disciplinaire...), lisible (compréhensible par tous), surreprésenté (en raison de son étouffement systématique par la structure même de la prison) et mobile (ses «travailleurs » doivent pouvoir circuler dans la prison pour aller à la rencontre de tous les détenus) (de Thier et al., 2005).

Fragmentées, ces luttes privilégient donc néanmoins certains bastions de l'arbitraire carcéral pour mener ses actions, parmi lesquels les transferts arbitraires et l'isolement individuel. Il s'agit alors de mener des actions auprès des tribunaux administratifs au nom de détenus individuels. L'objectif est de « faire avancer la jurisprudence en attaquant des mesures d'ordre interne sur lesquelles se fonde l'arbitraire carcéral».

Une connexion, essentielle pour notre analyse, entre les subjectivations des détenus et la lutte juridique réside dans le fait que les détenus qui déposent des recours devant les tribunaux sont également ceux qui "prennent la parole " (subjectivation politique au sens de Rancière) et qui fomentent tout un ensemble de résistances de type infrapolitique (rébellion, protestation interne, négociation, pression, etc.). Parmi les pétitionnaires de Clairvaux, par exemple - voir notre introduction - figurent aussi des experts en droit. Notre interlocuteur insiste : ce sont très souvent « les mêmes détenus » qui mobilisent le droit et font avancer la jurisprudence. Il s'agit alors d'une part d'accompagner et d'aider les détenus qui n'ont pas d'avocat, mais également de soutenir régulièrement ces détenus particulièrement activistes.

Il y a un certain nombre de détenus, aussi, qui existent par ça, qui vivent leur détention grâce à ça. Si ils sont pas complètement écrasés par l'Administration pénitentiaire, c'est parce que les types ils se battent devant les tribunaux. T'en as un certain nombre qui sont comme ça. En général, on sait lesquels c'est, c'est des types qui sont en DPS [détenus particulièrement signalés], à qui l'A. P. en fait voir de toutes les couleurs, et la façon de se battre pour eux... Bon, il y en a qui accumulent les tentatives d'évasion, etc., ce qui les empêche pas de présenter des recours par ailleurs... mais il y en a d'autres qui arrivent à se maintenir en face de l'administration pénitentiaire, à se maintenir en face d'elle dans une situation d'égal à égal je dirais, parce que quelqu'un qui résiste à l'arbitraire, à la contrainte, par le droit, il sera... Il se sentira armé d'une certaine manière, même si il traverse des hauts et des bas... Mais on voit que pour eux, c'est important.

2.2. Un réseau hétérogène qui s'alimente et se soutient mutuellement

L'activité de ce cause lawyer nécessite de s'appuyer sur l'activité de toute une série d'acteurs et d'institutions qui crédibiliseront les revendications, solidifieront les arguments juridiques déployés et légitimeront son action. Ainsi, le commissaire aux droits de l'homme ou les travaux de la Commission Nationale Consultative des Droits de l'Homme (CNDH) fournissent un ensemble de préconisations précieuses sur lesquelles s'appuyer $^{15}$. Les instances supranationales jouent également un rôle crucial. Ainsi, le Comité de prévention de la torture (CPT), la Cour européenne des droits de l'homme (CEDH) et le Conseil de l'Europe forment un triptyque institutionnel porteur de propositions dont l'existence même change partiellement les termes du débat public. 
Malgré la faiblesse structurelle des Règles pénitentiaires européennes formulées par le Conseil de l'Europe, celles-ci constituent désormais une nouvelle arme pour quiconque voudrait dénoncer, sur la base d'un texte légitimé par un vote unanime des 46 États membres, le régime et les conditions de détention d'un système pénitentiaire de l'un de ces États membres. Il s'agit donc d'une arme spécifiquement normative (par opposition ici à une arme proprement judiciaire) en ce sens qu'elle donne du grain - du grain légitime, européen, institutionnel - à moudre à l'entrepreneur moral ${ }^{16}$ qui entend changer des lois et des pratiques carcérales indignes d'États démocratiques. Par exemple, l'affirmation du réformateur Norman Bishop selon laquelle « il est de la responsabilité des États membres de donner suite aux propositions de la Recommandation » devient, grâce aux Règles ellesmêmes, performative; c'est dans cette logique que ces textes sont mobilisés. En bref, l'existence de ces recommandations intensifie les possibilités de mise à l'épreuve des discours, décisions, engagements et pratiques gouvernementales en matière pénitentiaire par les militants qui se saisissent du droit comme arme.

Plus important peut-être, parce que précisément elles s'inscrivent dans un réseau institutionnel lourd, ces Règles sont un tout petit peu plus que de simples recommandations. Ainsi, Françoise Tulkens, juge à la cour européenne des droits de l'homme, explique : «La Cour prend de plus en plus en considération ce qui est accepté dans les Règles pénitentiaires européennes. Elles sont importantes car elles traduisent un consensus européen. Ce consensus est décisif, car si la Cour avance de manière solitaire et aveugle, les États résistent et rien n'avance. La Cour fait également un usage de plus en plus fréquent des rapports du Comité européen de prévention de la torture (CPT), qui peuvent servir à étayer une plainte» (Tulkens, 2006). Ainsi, bien que la Cour avance lentement, pas à pas, et ce de manière à garantir sa sécurité juridique («faiblesse » qui est aussi la condition même de sa force, de sa solidité juridique), et bien que les modes de fonctionnement de chacune des institutions concernées ne soient pas identiques ${ }^{17}$, il existe bel et bien certaines formes de «passerelles " propices à instaurer une dynamique jurisprudentielle favorable à la défense des droits fondamentaux ${ }^{18}$, dynamique à laquelle les Règles sont, même modestement, partie prenante. Dans le même sens, les rapports du CPT peuvent, devant un tribunal, constituer des éléments de preuve pour dénoncer des pratiques, ou servir de levier pour faire basculer une jurisprudence.

L'appui des médias est jugé lui aussi essentiel; il publicise une situation intolérable, même si le recours au droit est perdu d'avance. Ainsi, l'OIP peut profiter d'un recours pour proposer un article à la presse qui dénonce une situation intolérable, et, précisément, cette dénonciation aura d'autant plus de force et de légitimité qu'elle sera «blindée » d'un point de vue juridique.

Moi, je pense que ça se combine assez facilement... Les fois où on fait un communiqué, ça marche pas mal. La dénonciation publique, normalement, ça devrait à chaque fois se combiner. Parce que tu peux échouer, mais ça t'empêche pas de... T'as ouvert ta gueule une première fois, ça n'a pas marché, mais tu continues de l'ouvrir, et tu as toujours la possibilité de dénoncer, et ça te donne une bonne fenêtre médiatique pour le faire, et puis ça percute davantage dans l'esprit des gens. Malgré tout, on est quand même dans une société où on considère que l'administration doit se plier à la règle de droit.

Plus généralement, on retrouve les ingrédients présents dans des mouvements de cause lawyers plus anciens et plus installés. Aux États-Unis par exemple, Anthony Romero, directeur exécutif d'ACLU (American Civil Liberties Union) identifie trois stratégies nécessairement conjointes: l'activité proprement judiciaire et l'action devant les 
tribunaux pour faire respecter les droits bafoués, le travail législatif qui vise à promouvoir des lois plus justes ${ }^{19}$, et un « programme de communication et d'éducation du public ». Anthony Romero explique ainsi « vous pouvez gagner au Congrès ou au niveau des États, vous pouvez gagner au tribunal, mais si vous ne changez pas le cœur ou l'esprit des gens, vous aurez beau gagner deux ou trois batailles, à long terme vous perdez la guerre. C'est à mon avis ce qui est souvent arrivé au mouvement de défense des droits civiques aux États-Unis» (Romero, 2006). Notre interlocuteur s'inscrit de fait dans la continuité de cette triple stratégie, en montrant comment l'état des sensibilités collectives pèse sur les décisions du Conseil d'État.

Par exemple sur les matelas, on a agi en contentieux, les matelas toxiques qui dégagent un gaz. On est allés aussi dans les médias, régulièrement, en disant qu'il y a des matelas hautement toxiques, ce dont l'A.P. a parfaitement connaissance, etc., C'est obligé d'y aller de façon complémentaire parce que de toutes façons, tu sais très bien que le juge interne, au moins le Conseil d'État n'évolue que au moment où ils sentent la pression, le décalage de sa position par rapport aux attentes de la société relativement au fonctionnement d'une de ses institutions, etc. devient trop criant pour pouvoir rester sur le statu quo. Donc on a besoin de la presse. Ça se joue souvent aussi en partie... Tant que ça tient, qu'une position arbitraire tient, elle le fait, à partir du moment où ça devient trop dur, où il y a une critique dans la presse, dans les articles de doctrine et les risques de censure par la CEDH, là, ils anticipent et changent leur position.

Ce travail « d'éducation » concerne les juristes eux-mêmes, au premier rang desquels les avocats qui découvrent l'univers carcéral et les universitaires. Ainsi, par exemple, lorsqu'un avocat formé au droit pénal mais pas au droit pénitentiaire est amené à défendre un client au prétoire, il arrive qu'il demande conseil à l'OIP. De même, des efforts sont déployés afin d'engager des dialogues avec différents universitaires pour influencer directement le savoir sur les droits :

C'est vrai que bon, on a toujours la possibilité d'influencer, par l'intermédiaire des commentaires de doctrine, d'une décision, une dénonciation d'une réalité qui permet éventuellement au pouvoir législatif de réaliser les difficultés posées par telle ou telle question... Donc là, on essaie d'avoir un travail de... On peut appeler ça lobbying pédagogique, je sais pas suivant la façon dont on se place, mais en tout cas essayer d'alimenter un certain nombre de profs de droit en décisions, etc., et faire partager nos appréciations, etc., et qu'elles soient éventuellement reprises dans les commentaires... Tout ça, ça crée un corps de doctrine qui a quand même une incidence sur la façon dont l'A. P. voit qu'elle est perçue par l'extérieur et notamment par les praticiens du droit, et ça peut être franchement utile.

Enfin, certaines transformations juridiques peuvent très bien présenter des effets secondaires. Par exemple, l'arrivée de l'avocat au prétoire n'a pas véritablement permis de transformer les pratiques des commissions disciplinaires. Mais la présence de l'avocat, de fait, intensifie la présence d'un « œil extérieur » sur l'institution, qui peut alimenter en retour l'activité juridique :

Il arrive même que nous on ait des avocats au téléphone sur une affaire, et l'avocat saisit l'occasion du coup de fil pour dire « je sors d'une commission de discipline, j'ai vu ça aujourd'hui, c'était franchement louche, etc. ». C'est déjà arrivé, même si peu sont ceux qui spontanément se tournent vers nous pour nous faire savoir un dysfonctionnement.

Ce rapide survol de la manière dont se structure le cause lawyering en matière carcérale, en indique à lui seul l'enjeu intellectuel : il est impossible de cantonner l'analyse de la progression et des usages du droit en prison dans une seule perspective juridique : c'est 
bien d'une sociologie de l'usage du droit dont nous avons besoin ici. Impossible enfin de se cantonner à la critique de l'inertie de l'institution. Le survol de ces pratiques suffit à suggérer l'émergence d'une nouvelle police institutionnelle dont la condition même est d'être mise à l'épreuve par le droit, mise à l'épreuve juridique qui est alimentée par d'autres formes d'action: dénonciation, médiatisation, et également subjectivation politique.

51 Dans le réel, l'univers de la police et celui de la politique tels que l'envisage conceptuellement Rancière ne sont donc pas étanches. D'un point de vue théorique, ces réseaux du contre-pouvoir ainsi survolés mettent largement à mal la "pureté » du politique tel que l'envisage Rancière. On rejoint là très largement la critique de Nordmann: la politique ne peut-elle pas avoir aussi pour objet, et peut-être de façon essentielle, d'élaborer de nouvelles polices? «À renoncer à cette visée, on risque de s'enfermer dans un mode d'action exclusivement symbolique, hypotéquant ainsi l'efficacité des démonstrations d'égalité que l'on entend mener (...) Il n'est pas d'autre moyen pour s'assurer une "police» moins mauvaise que d'exiger, par des actions politiques, sa réforme (...) Si l'un des effets majeurs de la politique est de parvenir à transformer et à informer la police, il est pour le moins problématique de soutenir que la politique est essentiellement différente de la police » (Nordmann, 2006, 139-140).

\subsection{L'innovation comme devenir}

L'innovation constitue moins le produit d'une transformation singulière qu'un devenir, une aspiration contre l'intolérable à partir de laquelle peuvent s'ordonner les luttes concrètes. Explicitons davantage ici l'horizon utopique qui organise les luttes concrètes qui viennent d'être explorées, en retournant la perspective et les évidences qui structurent généralement la pensée pénitentiaire. Comment penser le développement d'une configuration de police carcérale qui retourne l'ordre des priorités? L'organisation guerrière-défensive de l'institution ne constituerait plus le butoir de l'avancée des droits ; au contraire, les droits des personnes incarcérées constitueraient le butoir inaliénable des principes sécuritaires de l'institution. Il s'agit de penser, en compagnie de Baratta et de Chauvenet, un approfondissement de la notion de sécurité, susceptible non plus de fragiliser la vie en prison, mais de renforcer une sécurité fondamentalement redéfinie comme le résultat d'une « conflictualisation » (se substituant à la « violence ») de la vie en prison.

53 Au lieu d'être consacrés en-deça de la limite de la sécurité, il paraît pertinent de penser les droits comme l'instrument d'une politique de sécurité « intégrale » soit une politique de « sécurité des droits » (Baratta, 1999). Baratta propose cette piste dans le champ de la politique criminelle. Il ne paraît pas inopportun d'en transférer les intuitions dans le champ carcéral. Baratta propose en effet une transformation épistémo-politique de la conception même de la politique criminelle, afin d'éviter qu'elle se substitue à la politique sociale dans un cadre contemporain qu'il caractérise par son efficientisme (soit la dépolitisation des questions criminelles et pénales au profit d'un pur calcul d'efficience). La sécurité est un concept à redéfinir comme « jouissance et (...) protection effective des droits fondamentaux contre toute atteinte ou traitement non approprié venant de personnes qui exercent une forme quelconque de pouvoir de fait ou de droit dans un espace territorial » (Baratta, 1999, 241). La sécurité, écrit-il encore, correspond au besoin d'être et de se sentir sûr dans l'exercice de tous les droits. Cette redéfinition fait de la sécurité des droits l'objet par excellence de la politique, en particulier dans les espaces territoriaux où cette sécurité est loin d'être aménagée. Ainsi, l'insécurité en prison relève 
bien plus de l'action ou de l'inaction du « pouvoir » que de l'action démunie des détenus. De ce point de vue, la suspension des droits justifiée par la sanction disciplinaire ou par l'impératif sécuritaire constitue logiquement la suspension de la sécurité elle-même (telle que définie par Baratta). Si l'on peut lire dans ces développements un programme de fragilisation de la prison, la perspective dialectise cependant l'apparent paradoxe de sa définition et son effet de fragilisation, puisqu'une " politique de justice sociale et d'égalité ne peut avoir que des conséquences positives sur le contrôle » (Baratta, 1999, 251) de la vie carcérale. Autrement dit, la protection des droits ne s'oppose pas à la sécurité ; elle est la principale composante de cette dernière.

Chauvenet nous explique que la prison est fragile et qu'elle n'est pas une institution, parce qu'elle n'institue rien. Cette fragilité est une condition de sa violence. À l'inverse, la fragilisation de la prison par une politique de sécurité intégrale, soit de sécurité des droits, est paradoxalement la condition de son institutionnalisation, de sa transformation en espace de droit et de droits. «La prison, comme la société tyrannique, est une structure labile, instable, imprévisible, ce qui constitue une des causes essentielles des violences qui ont lieu en son sein. Un des traits de la tyrannie est sa fragilité " (Chauvenet, 2006). Paradoxalement c'est dans la défaillance et l'inconsistance radicales de l'administration qu'apparaît le plus nettement et le plus cruellement la capacité sociale et politique des détenus eux-mêmes. Un article publié dans le Courrier International (novembre 2005) sur une prison du Malawi révèle comment, dans le dénuement et la surpopulation extrêmes, les détenus prennent en charge eux-mêmes et "civilement " leur sécurité, le règlement de leurs conflits et leur santé. L'imprévisibilité, quand elle ne s'attaque pas à la survie, porte notamment sur les règles. Instaurer des règles plus prévisibles, dont l'application puisse être l'objet de revendication par les détenus, constitue une perspective de fragilisation accrue de la prison, qui l'institutionnalise et dont les détenus deviennent les sujets politiques. La labilité des règles sert la tyrannie même assouplie; la rigidification des droits des détenus fait porter l'incertitude de l'action et de ses résultats tant par la prison que par le détenu lui-même. L'innovation se tient plus dans cette potentialité transformatrice que dans la reconnaissance des droits comme telle. Deux concepts sont intimement associés à cette représentation innovante des droits : la production d'une situation d'incertitude (quant à la structure et au programme de la prison, devenant espace « incertain » parce qu'espace de droit) et la constitution du détenu en sujet politique susceptible de mettre l'institution "à l'épreuve", soit (en paraphrasant Linhart et Moreau de Bellaing, 2005, 279) de lui faire vivre une situation au cours de laquelle une incertitude apparaît sur les qualités des protagonistes, des dispositifs et des régimes de l'institution carcérale, jusqu'à la stabilisation de leur identité ou la remise en cause de leurs attributs. Dans cette perspective, l'enjeu de la promotion des droits des détenus est de constituer les détenus en sujets politiques, capables d'organiser la vie en détention. Le rapport juridique ainsi «normalisé »-c'est-à-dire extrait des contraintes pénologiques et sécuritaires qui en limitent jusqu'à la juridicité responsabilise le détenu comme sujet politique (et non comme assujetti) et fragilise la prison en rendant incertaines les conditions despotiques de son fonctionnement (autrement dit en accroissant, par le droit, les exigences de justification de son fonctionnement). Les épreuves subies par la prison deviennent sous cet angle des épreuves réellement politiques en tant qu'elles font peser l'incertitude autant sur la prison que sur le détenu. Le rapport juridique est en effet le rapport moderne d'une conflictualisation dont il est clair qu'elle est impossible aujourd'hui en prison; de cette impossibilité naît la violence (Chauvenet, 2006). 

portée fondamentale et innovatrice du concept de sécurité dynamique, soit une sécurité fondée sur le dialogue et le conflit et non sur l'autoritarisme, la sécurité statique et la violence. Si l'on en perçoit déjà les instrumentalisations, il n'empêche que la proposition de Baratta constitue la base d'une innovation gouvernementale: en quelque sorte, l'instrumentalisation des droits comme processus de subjectivation constituera ou constitue déjà l'enjeu de la lutte suivante dans la dialectique infinie de la politique et de la police. Cet enjeu a bien été saisi lors de la campagne pour les «États généraux de la condition pénitentiaire » puisqu'il s'agissait de faire émerger, à partir des cahiers de doléances des détenus eux-mêmes (issu d'un questionnaire auquel les détenus ont massivement répondu), les réformes les plus urgentes. Cet enjeu - la restitution au détenu de son statut de sujet politique - rappelle à tous les réformateurs carcéraux l'actualité du vieux proverbe touareg : « Tout ce qui est fait pour nous, sans nous, est contre nous".

Conclusion : la politique des droits, une lutte vivante et incertaine

56 La fragilisation de la prison, par l'instauration d'un garantisme positif, forme la base structurale sur laquelle devient pensable la transformation de la violence en conflit et de l'insécurité en incertitude. Au delà de cette suggestion spéculative pour une police carcérale radicalement innovante et, conséquemment, moins indigne, il faut rappeler la légitimité ultime du surgissement démocratique dans une logique policière donnée, qui interdit de cantonner la réflexion sur l'innovation à la proposition d'une autre configuration, fût-elle le support d'un renversement de tendance respectueux des « droits de l'homme».

Les temps de la démocratie militante ont décliné toute une série de formes polémiques des «hommes nés libres et égaux en droit». Des «nous» ont pris différents noms de sujets pour éprouver la puissance litigieuse des «droits de l'homme ", pour mettre à l'épreuve l'inscription de l'égalité, demander si les droits de l'homme étaient plus ou moins que les droits du citoyen, s'ils étaient ceux de la femme, du prolétaire, de l'homme noir et de la femme noire, etc. Ils ont ainsi donné aux droits de l'homme toute la puissance qu'ils peuvent avoir: la puissance de l'inscription égalitaire augmentée par celle de son argumentation et de sa manifestation dans la construction du cas de litige, dans la mise en rapport du monde de validité de l'inscription égalitaire et de son monde de non-validité. Le règne de l'« humanitaire » commence en revanche là où les droits de l'homme sont coupés de toute capacité de singularisation polémique de leur universalité, où la phrase égalitaire cesse d'être phrasée, interprétée dans l'argumentation d'un tort qui manifeste son effectivité litigieuse. Alors l'humanité n'est plus attribuée polémiquement aux femmes et aux prolétaires, aux Noirs ou aux damnés de la terre. Les droits de l'homme ne s'éprouvent plus comme capacité politique. Le prédicat «humain » et les «droits de l'homme » sont simplement attribués, sans phrase, sans médiation à leur ayant-droit, le sujet « homme ». L'ayant-droit pur et simple n'est alors pas autre chose que la victime sans phrase, figure dernière de celui qui est exclu du logos, muni seulement de la voix exprimant la plainte monotone, la plainte de la souffrance nue, que la saturation a rendue inaudible (Rancière, 1995, 171-172).

57 Le carcéralo-centrisme cherche à réaliser ce qu'avec Rancière on peut appréhender comme le paradoxe de la suppression politique de la politique. Cette annulation a un nom : le « consensus », saisi comme annulation du dissensus, réduction de la politique à la police. Le consensus tend à faire disparaître le fondement de la politique selon Rancière : l'émergence de sujets politiques manifestant face aux autres leurs désaccords, sous la 
forme d'un conflit révélant - même tendanciellement - sa portée universelle. C'est cette dynamique et cette "portée universelle » qui font, selon nous, et ce au delà de sa domestication contemporaine, de la question des droits des détenus une lutte vivante. La dynamique critique de l'idée démocratique ne cherche pas d'état stable. Elle est partiellement "récupérable » en tant qu'elle peut favoriser l'innovation pénale, soit l'émergence d'une nouvelle police de la peine et de la prison); elle est également " irrécupérable ", dans le sens où son essence même est le dissensus, le litige ouvrant sur une dialectique de la politique et de la police.

La véritable innovation ne relève pas de la découverte fort improbable d'un «bon gouvernement de la prison" mais de la création, par la politisation de son fonctionnement, des conditions pour une forme de gouvernement incertaine. La rationalité et l'appareillage pénitentiaires, comme l'État démocratique, se soumettent, dans cette perspective, à une exigence d'affaiblissement légitime, plutôt qu'à un programme axé sur leur relégitimation. La fragilisation de la prison tient au double critère de l'émancipation $d u$ sujet politique que constitue le détenu et du décloisonnement (ou de l'accroissement de la porosité) de la prison (Kaminski, 2002). Mais la légitimité ultime du surgissement démocratique dans une logique policière donnée, interdit de marquer du sceau de l'innovation la proposition d'une autre configuration, fût-elle le support d'un renversement de tendance, le concept restant définitivement, dans la logique démocratique, marqué du sceau de l'incertitude. Un tel enjeu, si on le comprend bien, rend antithétique l'énoncé selon lequel «les détenus doivent se constituer en sujets politiques ", puisqu'une telle constitution est par nature indéterminée. L'indétermination démocratique est la condition même de l'existence du sujet politique et de la perpétuation de la dialectique police/politique. Le surgissement de la démocratie en prison doit ainsi être appréhendé dans ce cadre conceptuel comme une entreprise de politisation qui cherche à interrompre l'ordre de la domination structurelle dont les détenus sont l'objet en perturbant, par le biais même de cette politisation, la logique policière qui sous-tend, stabilise et légitime la structure de l'institution. La lutte est singulière, mais elle s'outille sur l'universalité de son arme. Elle fait jouer polémiquement l'universel - historique ${ }^{20}-$ et le particulier. La vérité des droits de l'homme ici n'est pas indexée à une qualité universelle et a-temporelle mais à l'historicité des structures politiques et de leurs représentants incarnés, tels la pénalité et en particulier la prison, tels les droits de l'homme et en particulier ceux du détenu.

\section{BIBLIOGRAPHIE}

Artières P., Quéro L., Zancarini-Fournel M. (Documents réunis et présentés par), 2003, Le groupe d'information sur les prisons. Archive d'une lutte 1970-1972, Paris, IMEC.

Baratta A., 1999, Droits de l'homme et politique criminelle, Déviance et société, 23, 3, 252-253.

Becker H.S., 1985 [1963], Outsiders, Études de sociologie de la déviance, Paris, Métailié.

Beernaert, M.-A., 2007, Manuel de droit pénitentiaire, Louvain-la-Neuve, Anthemis, coll. Criminalis. 
Bérard J., Chantraine G., 2006, Nous, les emmurés vivants, Vacarme, 38, 52-56.

Bérard J., Chantraine G., 2007, Ai-je le droit d'avoir des droits ?, Vacarme, 40, 52-55.

Bishop N., 2006, La participation des personnes détenues à l'organisation de la vie en détention (avril), Champ Pénal / Penal Field, http://champpenal.revues.org/document485.html.

Boltanski L., 1990, L'amour et la justice comme compétences, Paris, Métailié.

Bouloc B., 2005, Opportunité d'une loi pénitentiaire... pour les détenus, Revue pénitentiaire et de droit pénal, 1, mars, 37-61.

Cartuyvels Y., 2002, Réformer ou supprimer : le dilemme des prisons, in De Schutter O., Kaminski D. (Eds.), L'institution du droit pénitentiaire Paris/Bruxelles, LGDJ/Bruylant, La pensée juridique, 113-132.

Cauchie J.-F., 2005, Un système pénal entre complexification et innovations. Le cas ambivalent des travaux communautaires belges, Déviance et société, 3, 399-422.

Cauchie J.-F., Kaminski D., 2007, Éléments pour une sociologie du changement pénal en Occident. Éclairage des concepts de rationalité pénale moderne et d'innovation pénale, Champ Pénal / Penal Field, mis en ligne le 14 mai. URL : http://champpenal.revues.org/document613.html.

Céré J.-P., 2002, Les droits des détenus sous la $\mathrm{V}^{\mathrm{e}}$ République : de réels progrès ? Revue française d'administration publique, 99, 417-426.

Chantraine G., 2004a, Prison et regard sociologique : pour un décentrage de l'analyse critique, Champ penal/Penal Field, Vol. 1, http://champpenal.revues.org/document39.html.

Chantraine G., 2004b, Prison et regard sociologique Champ pénal / Penal Field, http:// champpenal.revues.org/document39.html.

Chantraine, G., 2006a, La prison post-disciplinaire, Déviance et Société, 30, 3, 273-288.

Chantraine G., 2006b, Du progrès carcéral, Vacarme, 36, 15-19.

Chantraine G., Vacheret M., 2005, Expertise psychologique, gestion des risques et rapports de pouvoir dans les pénitenciers canadiens, Questions Pénales, septembre.

Chauvenet A., 2006, Privation de liberté et violence : le despotisme ordinaire en prison, Déviance et société, 30, 3, 373-388.

CNCDH, 2007, Sanctionner dans le respect des droits de l'homme, Paris, La Documentation Française, 2 vol.

Cohen S., 1985, Visions of Social Control, Cambridge, Polity.

Comité éditorial, 2005, À l'épreuve du scandale, Politix, 18, 71, 4.

De Blic D., Lemieux C., 2005, Le scandale comme épreuve. Éléments de sociologie pragmatique, Politix, 18, 71, 9-38.

de Thier F., Lebrun,V., Spronck V., 2005, Murmures d'une prison : cela en vaut-il la peine ?, La prison, droit dans le mur ? Colloque du $\mathrm{XXV}^{\mathrm{e}}$ anniversaire de l'Association syndicale des magistrats, Bruxelles, 28 janvier, non publié.

Dobry M., 1992, Sociologie des crises politiques. La dynamique des mobilisations intersectorielles, Paris, presses de la FNSP, coll. Références.

Dünkel F., 1997, La position en droit du détenu et les possibilités de contrôle légal des décisions relatives à l'exécution des peines en Allemagne, in Société belge de Criminologie, Position en droit 
et droit de plainte du détenu, Les Dossiers de la Revue de droit pénal et de criminologie, Bruxelles, La Charte, 2, 17-36.

Enguéléguélé S., 2002, Création de la loi pénale et analyses des politiques publiques, in Mucchielli L., Robert Ph. (dir.), Crime et sécurité, l'état des savoirs, Paris, La Découverte, 76-83.

Foucault M., 1994a [1979], Luttes autour des prisons, Dits et écrits, Paris, Gallimard, t. III, 806-818.

Foucault M., 1994b, Omnes et singulatim : vers une critique de la raison politique, Dits et écrits, Paris, Gallimard, t. IV, 134-161.

Foucault M., 2001 [1982], Le sujet et le pouvoir, Dits et écrits II, 1976-1988, Paris, Gallimard, Quarto, 1041-1062.

Frigon S., 2002, La création de choix pour les femmes incarcérées : sur les traces du groupe d'études sur les femmes purgeant une peine fédérale et de ses conséquences, Criminologie, 35, 2, 9-30.

Hall P., 1986, Governing the Economy: the Politics of State Intervention in Britain and France, New York, Oxford University Press.

Hall P., 1993, Policy Paradigm, Social Learning and the State, the Case of Economic Policy in Britain, Comparative Politics, 25, 3, 275-296.

Hannah-Moffat K., 2005, Criminogenic needs and the transformative risk subject, Punishment and Society, 2005, 7, 1, 29-51.

Hannat-Moffat K., 2000, Prisons that empower, Neo-liberal Governance in Canadian Women's Prisons, British Journal of Criminology, 40, 510-531.

Herzog-Evans, M., 2002, La révolution pénitentiaire française, in De Schutter O., \& Kaminski D., (Eds.), L'institution du droit pénitentiaire, Enjeux de la reconnaissance des droits des détenus, Paris/ Bruxelles, LGDJ, La pensée juridique, 17-41.

Hughes E.C., 1975, Dilemmas and Contradictions of Status, American Journal of Sociology, 50, 353-359.

Kaminski D., 2002, Les droits des détenus au Canada et en Angleterre : entre révolution normative et légitimation de la prison, in De Schutter O., Kaminski D. (Eds.), L'institution du droit pénitentiaire. Enjeux de la reconnaissance des droits des détenus, Paris/ Bruxelles, L.G.D.J./Bruylant, La pensée juridique, 91-112.

Kaminski D., 2006, Un nouveau sujet de droit pénal ?, in Digneffe F., Moreau Th. (dir.), Responsabilité et responsabilisation dans la justice pénale, Bruxelles, De Boeck-Larcier, 45-64.

Lascoumes P., 1990, Pluralité d'acteurs, pluralité d'actions dans la création contemporaine des lois, in Coll., Acteur social et délinquance. Une grille de lecture du système de justice pénale, Liège/ Bruxelles, Mardaga, 145-163.

Lascoumes P., 2006, Ruptures politiques et politiques pénitentiaires, analyse comparative des dynamiques de changement institutionnel, Déviance et Société, 30, 3, 405-418.

Linhart D., Moreau de Bellaing C., 2005, Légitime violence ? Enquêtes sur la réalité de l'État démocratique, Revue française de science politique, 55, 2, 269-298.

Martuccelli D., 2006, Forgé par l'épreuve, Paris, Armand Colin.

Mary Ph., 1988, Révolte carcérale. Changements et logique pérenne de la prison, Bruxelles, StoryScientia.

Mary Ph., 2006, La nouvelle loi pénitentiaire. Retour sur un processus de réforme (1996-2006), Courrier hebdomadaire du CRISP, nº 1916. 
Mathiesen T., 1990, Prison on Trial, London, Sage.

Morgan R., 1994, Justice and Responsibility in Prisons, in Jones I.G., Williams G., (eds), Social Policy, Crime and Punishment. Essays in Memory of Jane Morgan, Cardiff, University of Wales Press, 107-123.

Nordmann Ch., 2006, Bourdieu/Rancière, La politique entre sociologie et philosophie, Paris, Éditions Amsterdam.

O’Malley P., 1992, Risk, Power and Crime Prevention, Economy and Society, 21, 252-275.

Pires A.P., 1992, La réforme pénale et la réciprocité des droits, Criminologie, 25, 1, 74-104.

Pires A.P.,1998, Aspects, traces et parcours de la rationalité pénale moderne, in Debuyst Chr., Digneffe Fr., Pires A.P. (eds.), Histoire des savoirs sur le crime et la peine. Vol. 2, Montréal-OttawaBruxelles, PUM-PUO-De Boeck Université, 3-52.

Quirion B., 2006, Traiter les délinquants ou contrôler les conduites : le dispositif thérapeutique à l’ère de la nouvelle pénologie, Criminologie, 39, 2, 137-164.

Rancière J., 1995, La Mésentente, Philosophie et Politique, Paris, Galilée, La philosophie en effet. Reynaert P., 2004, La prison entre immobilisme et mouvement perpétuel, in Kaminski D., Kokoreff, M., Sociologie pénale : système et expérience, Ramonville Saint-Agne, Érès, 235-255.

Robert D., Frigon S., 2006, La santé comme mirage des transformations carcérales, Déviance et Société, 30, 3, 305-322.

Romero A., 2006, Un civisme radical. Entretien avec Antony Romero, Vacarme, 34, 33-39.

Shaw M., Hannah-Moffat K., 2002, La contrainte des choix : un regard rétrospectif, Criminologie, $35,2,53-72$.

Sim J., 1994, The Abolitionist Approach : A British Perspective, in Duff A., Marshall S., Dobash R.E., Dobash R.P. (eds), Penal Theory and Practice, Manchester, Manchester University Press, 1994, 263-284.

Tulkens Fr., 2006, L'extension du domaine de droits (interview), Dedans-Dehors, 54, mars-avril, 24-27.

Tulkens Fr., van de Kerchove M., 2005, Les droits de l'homme : bonne ou mauvaise conscience du droit pénal ?, in Verbruggen F., Verstraeten R., Van Daele R., Spriet B (red.), Strafrecht als roeping, Liber Amicorum Lieven Dupont, Leuven, Universitaire Pers Leuven, vol. 2, 949-968.

Vasseur V., 2000, Médecin-chef à la prison de la Santé, Paris, Le-Cherche-Midi.

Vigour C., 2004, Réformer la justice en Europe. Analyse comparée des cas de la Belgique, de la France et de l'Italie, Droit et Société, 56-57, 291-325.

Weaver K., 1986, The Politics of Blame Avoidance, Journal of Public Policy, 6, 4, 371-398.

\section{NOTES}

1. Les auteurs remercient Hugues de Suremain pour leur avoir éclairé différents aspects du militantisme juridique en matière de prison.

2. Les droits comme innovations sont donc un retournement (ou un processus simultané à son processus contraire) bien décrit, dans leurs contributions à ce dossier de Champ Pénal/Penal Field, par Richard Dubé : les droits de l'homme ont dans un premier temps eu « l'effet pervers » de renforcer la rationalité pénale moderne « de l'extérieur »; 
maintenant, les droits de l'homme infléchissent au moins partiellement la rationalité pénale moderne « de l'intérieur » : à l'intérieur des organes de cette rationalité, on lutte contre elle au nom des droits. Par contre, Tulkens et van de Kerchove (2005) montrent comment la jurisprudence de la Cour européenne des droits de l'homme met la convention au service de l'activité pénale et de son renforcement, les droits de l'homme « exigeant » pour leur respect une pénalisation croissante.

3. Les États généraux de la condition pénitentiaire réunissent la Confédération générale du travail (CGT pénitentiaire), le Conseil national des barreaux (CNB), Emmaüs France, la Fédération nationale des associations d'accueil et de réinsertion sociale (FNARS), la Fédération nationale des unions des jeunes avocats (FNUJA), la Ligue des droits de l'homme (LDH), l'Observatoire international des prisons (OIP), le Syndicat des avocats de France (SAF), le Syndicat de la magistrature (SM), l'Union syndicale des magistrats (USM) et le Syndicat national de l'ensemble des personnels de l'administration pénitentiaire (SNEPAPFSU).

4. Nous sommes proches ici du sens de la notion de police telle qu'utilisé par les auteurs du XVII ${ }^{e}$ et XVIII ${ }^{e}$ siècle pour désigner tout ce qui concerne «l'homme » et son «bonheur ». Voir Foucault, 1994b.

5. On s'appuie là sur Bérard et Chantraine (2006), qui analysent plus minutieusement la condition particulière des « longues peines ", condition dont cette pétition est symptomatique.

6. Bishop, 2006. Dans le même sens, la règle 50 des Règles pénitentiaires européenne préconise que « Sous réserve des impératifs de bon ordre, de sûreté et de sécurité, les détenus doivent être autorisés à discuter de questions relatives à leurs conditions générales de détention et doivent être encouragés à communiquer avec les autorités pénitentiaires à ce sujet ».

7. En ce sens, notre contribution à la réflexion sur l'innovation pénale ne s'articule pas à la théorie des systèmes mais relève de la réflexion " politique ». Soulignons-le : cette inflexion « politique » de la réflexion n'a rien d'incompatible avec une lecture systémique, elle déplace simplement le curseur de l'observation afin, en retour, de l'alimenter. Il s'agissait de redonner toute sa place aux mouvements sociaux et à l'activité démocratique dans l'analyse parfois trop « froide » des transformations systémiques. Nul mystère, en suivant cette hypothèse, quant à « l'origine » de l'intrusion d'un élément imprédictible qui renouvellerait une rationalité gouvernementale-carcérale. Cet élément n'est que le produit incorporé d'une lutte politique dans une reconfiguration gouvernementale ; ce processus démocratique est lui-même intrinsèquement imprédictible puisque le processus qui le caractérise est celui d'une refiguration du sensible, et non simplement la recherche de son organisation fonctionnellement optimale.

8. Soit un projet séculaire de rectification par la prison des comportements déviants dont l'échec n'aurait d'égal que sa reproduction monotone.

9. Nous nous appuyons ici sur Chantraine, Vacheret, 2005, et Chantraine, 2006a et b.

10. Voir à cet égard les recherches récentes de D. Martuccelli (2006).

11. Contrairement à l'idée confuse qui imagine une coexistence possible entre une prison qui respecte les droits des personnes et une prison qui impose sa volonté de redressement, il faut dire la contradiction originaire entre la discipline et le droit. La finalité d'une transformation des personnes, dans un cadre contraint, impose un déséquilibre de pouvoir irrémédiable. (...) Ce jeu de forces peut receler des trésors de sophistications, de ruses, mais il ne peut sortir de sa structure initiale : le patient est placé 
dans un espace artificiel où tout doit concourir, avec ou contre lui, à cette substitution de volonté. [Par exemple], l'opposition de la discipline et de l'exercice du droit à l'intimité et à une vie de famille est flagrante : lorsque les réformateurs carcéraux débattent du degré d'isolement propre à permettre le retour sur ses actes d'un délinquant plein de remords, il est bien évident qu'ils décident dans un sens ou dans un autre de la fréquence nécessaire, ou non, des rencontres du prisonnier. Si l'isolement est jugé profitable à la méditation, et le contact avec les proches corrupteur, il est logique que les personnes chargées de l'amendement dosent les rapprochements avec la famille selon leur technique, et non selon le droit de la personne à rencontrer sa famille (Bérard, Chantraine, 2007, 51).

12. Selon l'expression utilisée par D. Robert et S. Frigon, dans Robert, Frigon, 2006. 13. On ne fera pas de l'ordre policier ainsi défini la nuit où tout se vaut. La pratique des Scythes crevant les yeux de leurs esclaves et celles des modernes stratégies de l'information et de la communication qui, à l'inverse, donnent à voir sans limite relèvent toutes deux de la police. On n'en tirera aucunement la conclusion nihiliste que l'une et l'autre se valent. Il y a de la moins bonne et de la meilleure police - la meilleure, au demeurant, n'étant pas celle qui suit l'ordre supposé naturel des sociétés ou la science des législateurs mais celle que les effractions de la logique égalitaire sont venues le plus souvent écarter de sa logique « naturelle 》(Rancière, 1995, 53-54). 14. Sur les révoltes de novembre 2005, voir l'article publié par J. Rancière, dans Libération, en date du 18 janvier 2006 ; sur les sans-papiers, voir le texte de J. Rancière, publié dans Mouvements, en mars-avril 1999, 134-135. Pour une critique de ces positions, voir Nordmann, 2006.

15. Voir les deux récents rapports de la CNCDH, 2007.

16. S'il est évident que la conceptualisation d'Howard Becker est originellement ironique et critique, sa fécondité heuristique nous permet cependant de l'utiliser dans un sens neutre. Retenons donc ici la définition la plus simple de Becker : « Les normes sont le produit de l'initiative de certains individus, et nous pouvons considérer ceux qui prennent de telles initiatives comme des entrepreneurs de morale » (Becker, 1985 [1963]), 171).

17. « Le CPT est en charge de la prévention, rend des rapports généraux, fait des recommandations et dialogue avec les États. Nous [la CEDH] avons une fonction judiciaire et répressive. Les exigences sont différentes, car la Cour doit rendre dans des cas particuliers des jugements entièrement fondés en droit et en fait », (Tulkens, 2006, 25). 18. Sur cette dynamique générale, citons une dernière fois $F$. Tulkens, à propos de la CEDH : « Dans un arrêt Selmouni contre France du 28 juillet 1999, la Cour a pour la première fois qualifié de torture des faits qui se sont passés dans un commissariat de police. L'arrêt précise que le niveau d'exigence croissant en matière de droits fondamentaux fait en sorte que des actes auparavant qualifiés de traitement inhumain et dégradant sont désormais qualifiés de torture et que des actes qui auparavant ne rentraient pas dans le champ d'application de l'article 3 y entrent. Cet arrêt est souvent utilisé pour justifier le fait qu'il faut progresser dans la protection des droits », F. Tulkens, 2006, 25.

19. Pour l'OIP, c'est le cas lorsque, lors des «États généraux de la condition pénitentiaire ", la manifestation a culminé dans la demande de la promulgation d'une véritable loi pénitentiaire à même de protéger les droits fondamentaux des détenus. 20. À cet égard, Mathiesen propose de bien comprendre les « victoires » qu'ont constitué les abolitions historiques : l'abolition de l'esclavage (par exemple) constitue une innovation ; elle a déplacé et renouvelé les expressions de la discrimination raciale : toute 
innovation est une partie d'un processus historique et, pour cette raison même, enjeu d'une lutte politique infinie et toujours renouvelée (Mathiesen, 1990, 159).

\section{RÉSUMÉS}

La sociologie contemporaine de la prison reste globalement sceptique quant à l'impact réel du développement des droits des détenus sur le fonctionnement des institutions carcérales. D'un côté, la consolidation relative des droits des détenus serait incapable de détruire la primauté sécuritaire de l'institution : des privilèges sont transformés en droits formels, mais les exceptions justifiées par la nécessité sécuritaire les retransforment en privilèges. D’un autre côté, cette consolidation ne permettrait pas d'en finir avec la vocation disciplinaire de l'institution, mais constituerait au contraire une source inattendue de sa revitalisation. Si cette critique double, dont on détaillera l'ossature générale, permet de saisir la force de l'inertie de l'institution, elle n'offre cependant pas de focale pertinente pour observer les usages concrets du droit en détention. Ouvrant, de manière exploratoire, une sociologie du cause lawering en matière carcérale, cette contribution décrit la manière dont différentes ressources juridiques sont mobilisées pour affûter la lutte contre l'arbitraire carcéral et renforcer simultanément sa légitimité sociale. Dans ce cadre, l'«innovation pénale », soit, ici, une prison qui respecterait l'ensemble des droits de l'homme, constitue moins le produit d'une transformation singulière qu'un devenir, une aspiration contre l'intolérable à partir de laquelle peuvent s'ordonner les luttes concrètes. Le constat empirique de l'effet d'entraînement réciproque de différents registres d'action (subjectivation, recours administratif, dénonciation médiatique) outille parallèlement une discussion d'ordre proprement théorique. Celle-ci consiste à démontrer que l'étanchéité mutuelle des concepts de police et de politique tels que forgés, en philosophie, par Jacques Rancière ne permet pas de saisir la dynamique complexe des luttes démocratiques contemporaines.

Contemporary prison sociology remains, for the most part, sceptical as to the real impact of prisoners' rights development on the management of carceral institutions. On one hand, the relative consolidation of prisoners' rights is unable to undermine the security imperative of the institution: privileges are transformed into formal rights, but exceptions justified by the security imperative retransform those rights into privileges. On the other hand, this consolidation does not put an end to the disciplinary mission of the institution but is, on the contrary, a source of its revitalization. This dual critique, whose general framework will be further detailed, allows one to understand the strong inertia of the institution. However, it doesn't offer a relevant framework to observe the concrete uses of the law during detention. Pioneering a sociology of cause lawering inside the prison, the present contribution explores the ways in which different juridical resources are mobilized to hone the political struggle against prison arbitrariness while simultaneously reinforcing its social legitimacy. Within this framework, "penal innovation", that is to say, in this specific case, a prison that would respect all human rights, constitutes less the result of a specific transformation than an becoming, an aspiration against the intolerable from which concrete struggles can be organized. The empirical observation of the ratchet effect between various types of action (political subjectivation, administrative appeal, media denunciation) nourishes an appropriate theoretical discussion. This discussion consists in demonstrating that the mutual exclusiveness of the concepts of "police" and "politique", as 
Jacques Rancière philosophically puts it, does not permit one to fully grasp the complex dynamics of contemporary democratic struggles.

INDEX

Keywords : Prison, penal innovation, prisoners' rights, correctionnalism, politics, Jacques Rancière.

Mots-clés : innovation pénale, droits des détenus, cause lawering, correctionnalisme, politique, Rancière (Jacques)

\section{AUTEURS}

\section{GILLES CHANTRAINE}

Gilles Chantraine est chargé de recherches au CESDIP - CNRS (France) et directeur éditorial de Champ Pénal/Penal Field chantraine@ceqsdip.com.

DAN KAMINSKI

Dan Kaminski est professeur à l'Université catholique de Louvain-la-Neuve (Belgique) dan.kaminski@crim.ucl.ac.be. 